\title{
No evidence for sexual dimorphism of facial width-to-height ratio in four large adult samples
}

\author{
LEFEVRE, C. E. ${ }^{1 *}$, LEWIS, G. J. ${ }^{2}$, BATES, T. C. ${ }^{3}$, DZHELYOVA, \\ M. ${ }^{1}$, COETZEE, V. ${ }^{4}$, DEARY, I. J. ${ }^{3}$, \& PERRETT, D. I. ${ }^{1}$
}

1. School of Psychology, University of St Andrews.

2. Sage Center for the Study of the Mind, Department of Psychological and Brain Sciences, University of California, Santa Barbara.

3. Department of Psychology, University of Edinburgh.

4. Department of Genetics, University of Pretoria, Pretoria, South Africa.

* Corresponding author: School of Psychology, University of St Andrews, St Mary’s Quad, South Street, St Andrews, KY169JP, UK.Cel37@st_andrews.ac.uk

\begin{abstract}
Sexual dimorphism in physical appearance may be an important cue in both intra- and inter-sex competition. Recently, the facial width-to-height ratio (fWHR) has been proposed as a novel sexually dimorphic morphologic measure, with men suggested to have a higher fWHR than women. Currently, however, the status of fWHR as a sexually dimorphic trait is unclear. Here we tested for sexual
\end{abstract}


dimorphism in fWHR, as well as in three additional, previously

reported, facial measures, in four (three Caucasian and one African)

independent samples. In three of the four samples, no significant sex differences in fWHR were observed. In one sample males showed a significantly lower (rather than higher) fWHR than females (this effect was no longer significant after controlling for Body Mass Index). By contrast, significant and large sex differences were observed in all four samples for each of the three previously validated facial metrics;

namely: (i) lower face/face height, (ii) cheekbone prominence, and (iii) face width/lower face height. These results provide strong evidence against the claim that fWHR, at least as measured from the surface of the face, is sexually dimorphic.

\section{Introduction}

Sexual dimorphism refers to phenotypic characteristics that differ between males and females of the same species (Barber, 1995). Much attention has focused on sexual dimorphism in facial structure (e.g. Penton-Voak et al, 2001; Perrett et al., 1998): men tend to have larger jaws and more prominent brow ridges compared to women (Enlow, 1982). Recently work has suggested that the facial width-to-height ratio (fWHR: the ratio of bizygomatic width to upper face height; see Figure la) is a sexually dimorphic facial dimension, independent of body size (Weston, et al., 2007). However, not all 
studies have observed this dimorphism (Ozener, in press). Here we test the hypothesis of sexual dimorphism in fWHR utilising four homogenous samples, across two ethnicities (White European and Black African). Additionally we test the validity of several other putatively sexually dimorphic facial features.

Sexual dimorphism may arise as a result of inter-sexual selection (Andersson, 1992), whereby certain characteristics are retained or even amplified because they are favoured by members of the opposite sex. Alternatively, intra-sex competition could also lead to sexually dimorphic traits if such traits provide a selective advantage through dominance in competition over mates (for review see Puts, 2010). In either model, then, establishing sex differences in facial morphology is of considerable importance. As noted above, the fWHR has been proposed as one such sexually dimorphic facial feature.

Initial support for sexual dimorphism in the fWHR of human faces was based on direct skull measures in a sample of 121 modern black African skulls (68 male; Weston et al., 2007). The sample varied in age at death from less than 1 year to 30 years, with only about half the sample being fully grown at time of death. These findings were replicated in a mixed-ethnicity photographic sample of 88 North American undergraduates (37 male: Carre \& McCormick, 2008). Subsequent studies identified a range of sexually-dimorphic behavioural traits linking to fWHR amongst males: these include 
aggression (Carre \& McCormick, 2008; although see Deaner et al, in press), self-reported power (Haselhuhn \& Wong, 2012), and dishonesty (Stirrat \& Perrett, 2010). It has also been shown to signal aggression and untrustworthiness to others (Carre \& McCormick, 2009; Stirrat \& Perrett, 2010).

Despite the considerable attention centred on hypotheses derived from models assuming that fWHR is sexually dimorphic, this dimorphism itself has not yet been well established. The two studies reporting a sex difference in human samples (Carre \& McCormick, 2008; Weston et al., 2007) possessed small sample sizes and, in the case of Carre and McCormick (2008), contained mixed ethnicities. With regard to the latter issue, African populations may differ from Caucasians in their face shape (Enlow, 1982) suggesting potential bias in mixed ethnicity samples. Importantly, a recent attempt to replicate the sexual dimorphism of fWHR (Ozener, in press) reported no significant sex difference of fWHR in a Turkish sample larger than those of the initial positive studies $(n=470)$.

\subsection{The Current Study}

Here we examined sex differences in fWHR in three adult European samples and one adult African sample. Additionally, we measured three other documented sexually dimorphic facial dimensions: (i) lower face/face height, (ii) cheekbone prominence, and 
(iii) face width/lower face height (see Penton-Voak et al., 2001 and Figure lb) which have previously been linked to reactive testosterone (Pound et al., 2009). These additional measures allowed us to establish that the samples used in this study could produce known sexually dimorphic characteristics and also to establish whether these metrics are associated with fWHR.

One Caucasian sample was photographed using 3D imaging which yields methodological advantages for this type of study: specifically, 3D photographs provide to-scale representations of a participant's head, removing potential measurement errors that may arise from artefacts of head posture, i.e. faces rotated with respect to the camera in the horizontal or vertical planes.

\section{Method}

\subsection{Participants}

Four samples of human facial photographs were analysed. In all samples, participants were photographed with standardised distance to the camera and lighting and were instructed to keep a neutral facial expression.

Sample 1: 99 female $($ mean age $=20.21$, age range $=18-25)$ and 46 male (mean age $=20.24$, age range $=18-27)$ Caucasian undergraduate students. Participants were photographed using a 
Figure 1. Examples of measures and measuring points used for morphometric calculations. Measure for fWHR (a): horizontal lines represent the distance between the upper lip and highest point of the eyelids (upper face height), vertical lines represent the maximum distance between the left and right facial boundary (bizygomatic width). fWHR was calculated as width divided by height.

Morphometric calculations (b): (i) Lower face/face height: c-b/a-b, (ii) cheekbone prominence: ef/g-h, and (iii) face width/lower face height: e-f/c-b.
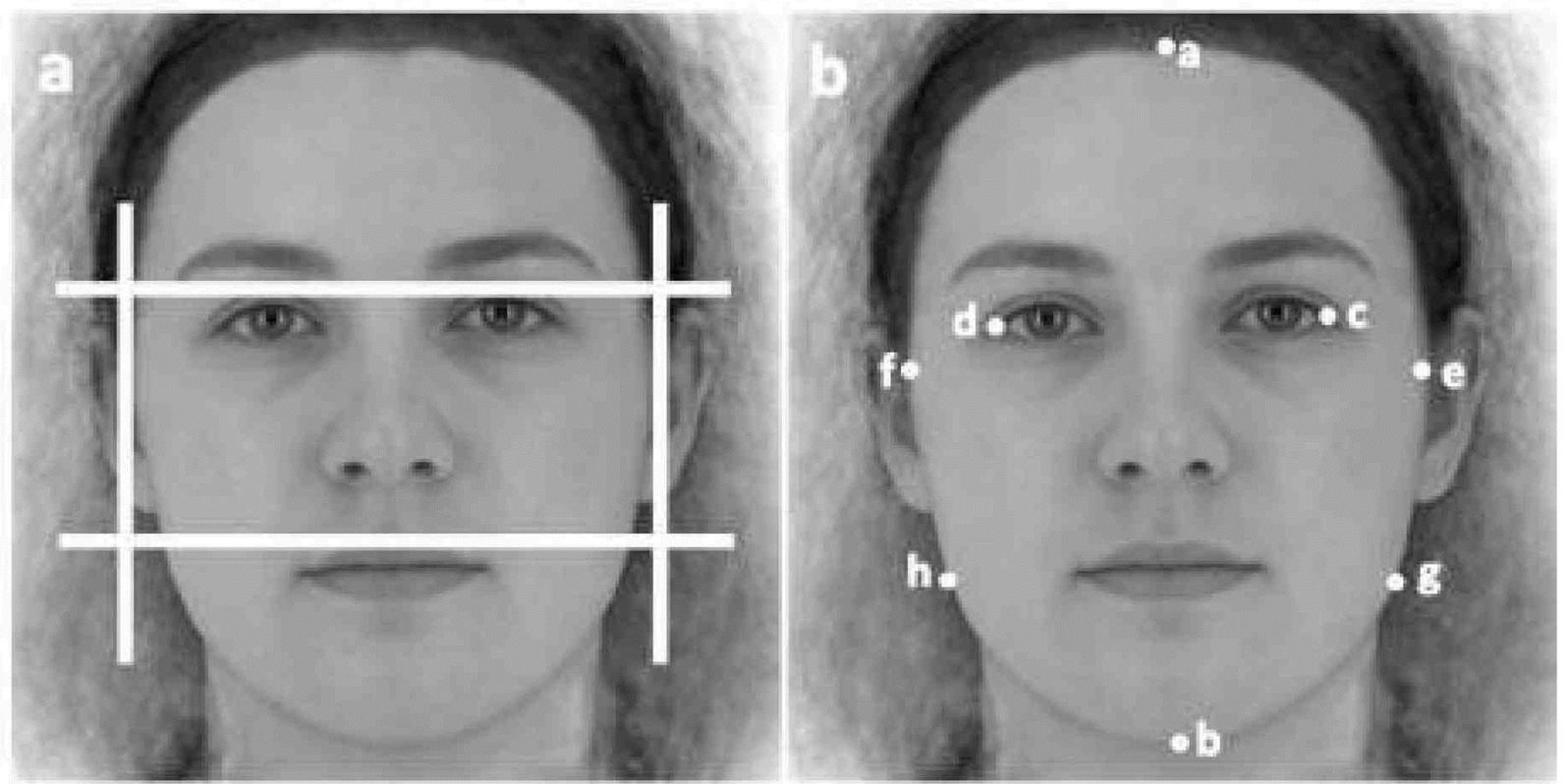
Fujifilm Finepix S5Pro digital camera.

Sample 2: 306 Scottish adults (169 female) from the Lothian Birth Cohort 1921 (Deary et al., 2004; Penke et al., 2009) who were all born in 1921 and approximately 83 years old - with a narrow range of ages - at the time of picture collection. Photographs were taken using a Nikon E5700 digital camera.

Sample 3: 124 male $($ mean age $=20.44$; age range $=18-30)$ and 131 female $($ mean age $=20.25$; age range $=18-28)$ Caucasian undergraduate students. Participants had 3D head model photographs taken using a 3dMD camera (for technical details see http://www.3dmd.com/3dmdface.html). Hair and clothing were occluded in all images before processing.

Sample 4: 110 female (mean age $=19.80$; age range $18-26$ ) and 108 male (mean age $=20.65$; age range 18-29) black South African undergraduate students. They were photographed using a Sony Cybershot DSC P72 or a Fujifilm Finepix S5Pro digital camera.

\subsection{Facial Measures}

Prior to measurement (using the Psychomorph software package; Tiddeman, et al., 2001, http://users.aber.ac.uk/bpt/jpsychomorph/), faces in all 2D samples were horizontally aligned and scaled to the same interpupillary distance. fWHR was measured by calculating the bizygomatic width 
(maximum horizontal distance from the left facial boundary to the right facial boundary) to upper-face height (vertical distance from the mid-point of the upper-lip to the highest point of the eyelids) ratio from photographs (see Figure la, and Stirrat and Perrett, 2010). For the 3D sample, measurements were made using Morphanalyser (Coetzee et al., 2011; http://cherry.dcs.aber.ac.uk:8080/wiki/MorphAnalyser). We also calculated facial metrics as described by Penton-Voak et al. (2001; see Figure lb); namely, (i) lower face/face height (vertical distance from mean eye height to gnathion/vertical distance from trichion to gnathion), (ii) cheekbone prominence (bizygomatic width/horizontal distance between left and right gonion approximation), and (iii) face width/lower face height (bizygomatic width/vertical distance from mean eye height to gnathion).

\section{Results}

Descriptive statistics for facial metrics in all four samples are shown in Table 1. The hypothesis that fWHR would be greater in male than in females was tested in each sample using independent t-tests, with fWHR as the dependent variable, and sex as the independent variable. Analysis revealed no significant sex differences for fWHR ii Samples 1,2, or 4, (all ps >.10); moreover, in all cases mean fWHR o women was in the opposite direction to that predicted (i.e., female 
Table 1: Sexual dimorphism in morphologic measurements. Sample 1: Caucasian undergraduate students; Sample 2: Scottish adults; Sample 3: Caucasian undergraduate students, with 3D photographs; Sample 4: South African black students.

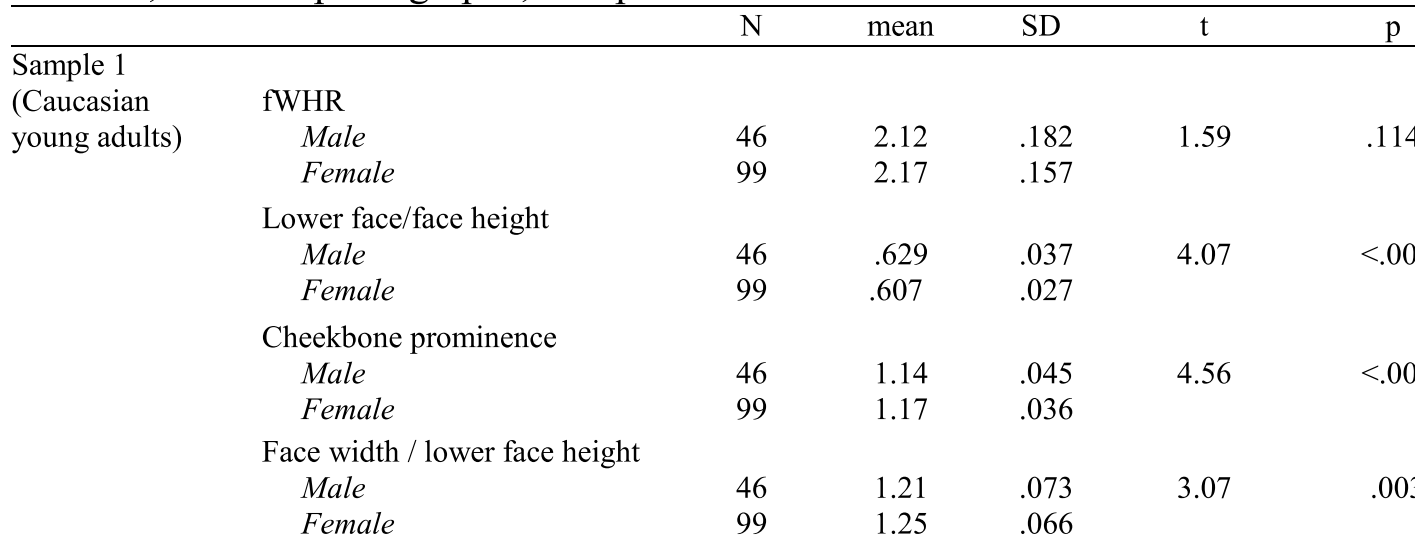

Sample 2

(Caucasian adults fWHR age 83)

\section{Male \\ Female}

Lower face/face height

Male

Female

Cheekbone prominence

Male

Female

Face width / lower face height Male

Female

Sample 3

(Caucasian young adults, 3D)

$$
\begin{aligned}
& \text { fWHR } \\
& \text { Male } \\
& \text { Female }
\end{aligned}
$$

Lower face/face height Male

Female

Cheekbone prominence Male

Female

Face width / lower face height Male

Female

Sample 4

(African young adults)
fWHR

$$
\text { Male }
$$

Female

Lower face/face height

Male

Female

Cheekbone prominence

Male

Female

Face width / lower face height

Male

Female

$\begin{array}{ccccc}137 & 2.06 & .170 & 1.47 & .142 \\ 169 & 2.09 & .164 & & - \\ \text { N/A } & - & - & - & \\ & & & & \\ 137 & 1.15 & .048 & 4.20 & <.001 \\ 169 & 1.18 & .055 & & \\ & & & & .019 \\ 137 & 1.25 & .067 & 2.37 & \\ 169 & 1.27 & .069 & & \end{array}$

1.84

.127

2.69

.008

124

131

.662

.114

5.18

$<.001$

124

.640

.025

1.14

.074

14.72

$<.001$

131

1.29

.086

$\begin{array}{lll}124 & 1.15 \quad .072\end{array}$

9.43

$<.001$

142

p

114

001

001

003 
fWHR was higher than that of men (see Table 1). In Sample 3, this female advantage in fWHR reached significance.

Slightly different nasion approximations have been used in the literature (Carre \& McCormick, 2008; Stirrat \& Perrett, 2010): We therefore re-analysed all faces following Carre \& McCormick's methods. These measures yielded slightly lower mean fWHR values but also showed no sex differences (all ps $>.10$ ).

Coetzee et al. (2010) report a positive association between fWHR and BMI. We therefore tested for effects of BMI on fWHR in Sample 1, 2, a subset of Sample 3 (91 males, 98 females) for whom BMI data were available, and in Sample 4 using ANCOVA (see Table 2). BMI was moderately correlated with fWHR in all samples (Sample 1: $\mathrm{r}=.27, \mathrm{p}=.001 ;$ Sample 2: $\mathrm{r}=.23, \mathrm{p}<.001 ;$ Sample 3: $\mathrm{r}=.40, \mathrm{p}<$ .001 ; Sample 4: $\mathrm{r}=.23, \mathrm{p}=.001)$. Sex differences remained non-significant for samples 1,2 , and 4 and became non-significant in sample 3 .

The other three face measures - lower face/face height, cheekbone prominence, and face width/lower face height - were highly sexually dimorphic in all samples (all ps $<.019$; see Table 1). Note that lower face/face height could not be calculated for the second sample (older adults) because the upper facial boundary could not be reliably determined in some of the men due to receding hairlines. 
Table 2: The effects of sex on fWHR, controlling for BMI and the interaction of sex*BMI, separately for each of the four samples.

\begin{tabular}{lcccc}
\hline Sample & $\begin{array}{c}\text { Overall } \\
\text { model }\end{array}$ & Sex & BMI & Sex*BMI \\
\hline & $\mathrm{F}$ & $\mathrm{F}$ & $\mathrm{F}$ & $\mathrm{F}$ \\
\cline { 2 - 5 } 1 & $4.79^{*}$ & 0.10 & $7.72^{*}$ & $<0.01$ \\
2 & $6.45^{* *}$ & 0.15 & $16.99^{* *}$ & 0.02 \\
3 & & & & \\
4 & $20.10^{* *}$ & 1.80 & $38.46^{* *}$ & 0.50 \\
\hline
\end{tabular}

Note. All results are from ANCOVA models with BMI as a continuous covariate.

Sample $1 \mathrm{df}=141$; Sample $2 \mathrm{df}=302$; Sample $3 \mathrm{df}=186$; Sample $4 \mathrm{df}=212{ }^{*} \mathrm{p}<.01$, $* * \mathrm{p}<.001$ 
The correlations between fWHR and width/lower face height was consistently positive (all $\mathrm{rs}>.399$, all $\mathrm{ps}<.001$ ). Lower face/face height was negatively associated with fWHR in Sample 1 (Caucasian young adults, $\mathrm{r}=-.233, \mathrm{p}<.001)$ and sample 3 (3D Caucasian young adults, $r=-.170, p=.007$ ), but positively in Sample 4 (African faces, $r=$ $.149, \mathrm{p}<.05)$. Cheekbone prominence was only associated with fWHR in Sample $3(\mathrm{r}=-.138, \mathrm{p}<.05)$.

\section{Discussion}

In the present study we tested whether facial width-to-height ratio (fWHR) and three other morphologic face measures are sexually dimorphic. In three of four tested samples of both Caucasian and African individuals we found no evidence for a greater fWHR in men than women. Moreover, and in contrast to recent work, in a 3D-head-model sample (Sample 3), men had a significantly lower fWHR than women, with all other samples showing the same directional trend; however, the effect in the 3D faces disappeared when controlling for BMI. In the other samples, controlling for BMI did not affect the results, although BMI itself was significantly (positively) associated with fWHR in each sample tested.

We assessed three other previously reported sexually dimorphic face measures: lower face/face height, cheekbone prominence and face width/lower face height. These measures yielded 
reliable sex differences in the direction expected from prior research (Penton-Voak et al., 2001; Pound et al. 2009), for each of our four samples. The correlations between fWHR and these three metrics were not straightforward. Face width/lower face height was positively associated with fWHR in all samples in contrast to the negative association expected if fWHR relates to facial masculinity.

Furthermore, while lower face/face height was positively (as expected) associated in the African sample the association was negative in Caucasian samples 1 and 3. Finally, cheekbone prominence was negatively associated with fWHR (as predicted) in only one of our samples (Caucasian 3D), with no association in the other samples. Hence facial width to height ratio is not consistently associated with other measures morphological of masculinity in facial structure.

The size and homogeneity of the four assessed samples in the current study may, at least in part, explain why results presented here differ from some earlier work. Previous studies reporting sexual dimorphism of fWHR (Carre \& McCormick, 2008; Weston et al., 2007) were conducted in relatively small samples vulnerable to influences from sampling bias and sample specific results. Several factors could cause spurious sex differences in facial measures, including BMI and ethnicity. BMI is positively associated with fWHR (Coetzee et al., 2010) and an unequal distribution of BMI between 
sexes may potentially cause apparent sex differences in this measure. Ethnic effects for fWHR may also exist in line with work demonstrating differences in facial bone structures between ethnic groups (Enlow, 1982). Accordingly, mixed ethnicity samples (as with Carre \& McCormick, 2008) could also affect results.

The present results give rise to the question why fWHR apparently associates with a range of sexually dimorphic behaviours (e.g. aggression and dominance in men: Carre \& McCormick, 2008; Stirrat \& Perrett, 2010) yet itself is not sexually dimorphic. One possible answer comes from research into facial soft tissue distribution. This work highlights that women, even when controlling for BMI, have greater facial adiposity, especially around the cheeks, than men (Enlow, 1982). This difference in facial adiposity may potentially conceal a sex difference in bone structure: men may have higher bizygomatic width than women, but this difference may not be apparent in 2D or 3D face measures because sex differences in facial adiposity obscure or reverse differences in fWHR when measured from the skin surface. As such, facial metrics measured on the surface may reflect the underlying bone structure more accurately in males than females due to men's lower facial adiposity. This, then, might explain why fWHR is related to behavioural traits in men (e.g. Carre \& McCormick, 2008; Haselhuhn \& Wong, 2012; Lewis et al., 2012; Carre et al., 2009; Stirrat \& Perrett, 2010; Stirrat \& Perrett, in press) 
but apparently not in women (Haselhuhn \& Wong, 2012). It should be noted, however, that recent work by Stirrat, Stulp and Pollet (in press), assessing fWHR directly from skulls, also found no mean sex difference in a large sample $(\mathrm{n}=862)$ suggesting alternative explanations may be required.

In summary, the present study strongly suggests that there are no sex differences between males and females in fWHR as measured on the surface of the face in either Caucasian or African populations. We did, however, successfully replicate previous work reporting sexual dimorphism in several other facial structures. These findings have implications for claims about sexual selection and behaviours believed to be associated with factors underlying facial structures and in particular the facial width-to-height ratio.

\section{Acknowledgements}

The Lothian Birth Cohort 1921 is supported by funding from the Chief Scientist Office of the Scottish Government (ETM/55). Ian Deary is supported by The University of Edinburgh Centre for Cognitive Ageing and Cognitive Epidemiology, part of the cross council Lifelong Health and Wellbeing Initiative (G0700704/84698). Funding from the Biotechnology and Biological Sciences Research Council (BBSRC), Engineering and Physical Sciences Research Council (EPSRC), Economic and Social Research Council (ESRC) and Medical Research 
Council (MRC) is gratefully acknowledged.

\section{References}

Andersson, M. (1992). Female choice for extreme tail length in a widowbird. Nature, 299, 818-820.

Barber, N. (1995). The evolutionary psychology of physical attractiveness: sexual selection and human morphology. Ethology and Sociobiology, $16,395^{\wedge} 24$.

Carre, J. M., \& McCormick, C. M. (2008). In your face: facial metrics predict aggressive behaviour in the laboratory and in varsity and professional hockey players. Proceedings of the Royal Society B-Biological Sciences, 275, 2651-2656.

Carre, J. M., McCormick, C. M., \& Mondloch, C.J. (2009). Facial Structure Is a Reliable Cue of Aggressive Behavior. Psychological Science, 20, 1194-1198.

Coetzee V., Chen J., Perrett D. I., \& Stephen I. D. (2010). Deciphering faces: Quantifiable visual cues to weight. Perception, 39, $51-61$

Coetzee, V., Re, D.E., Perrett, D.I., Tiddeman, B.P. \& Xiao, D. (2011). Judging the health and attractiveness of female faces: Is the most attractive level of facial adiposity also considered the healthiest? Body Image, 8(2), 190-193.

Deaner, R.O., Goetz, S.M.M., Shattuck, K., Schnotala, T. (in 
press). Body weight, not facial width-to-height ratio, predicts aggression in pro hockey players. Journal of Research in Personality.

Deary, I. J., Whiteman, M. C, Starr, J. M., Whalley, L. J., \& Fox, H.C. (2004). The impact of childhood intelligence on later life: Following up the Scottish mental surveys of 1932 and 1947. Journal of Personality and Social Psychology, 86, 130-147.

Enlow, D. (1982). Handbook of Facial Growth. Philadelphia: Saunders.

Haselhuhn, M.P., \& Wong, E.M. (2012). Bad to the bone: facial structure predicts unethical behaviour. Proceedings of the Royal Society B-Biological Sciences, 279, 571-576.

Lewis, G.J., Lefevre, C.E., \& Bates, T.C. (2012). Facial width-to-height ratio predicts achievement drive in US presidents. Personality and Individual Differences. Published online ahead of print: http://dx.doi.Org/10.1016/j.paid.2011.12.030

Ozener, B. (in press). Facial width-to-height ratio in a Turkish population is not sexually dimorphic and is unrelated to aggressive behaviour. Human Behavior and Evolution, DOI: 10.1016/j.evolhumbehav.2011.08.001.

Penke, L., Bates, T. C, Gow, A. J., Pattie, A., Starr, J. M., Jones, B. C, Perrett, D. I., \& Deary, I. J. (2009). Symmetric faces are a sign of successful cognitive aging. Evolution and Human Behavior, 30, 429-437. 
Penton-Voak, I.S., Jones, B.C., Little, A.C., Baker, S., Tiddeman, B., Burt, D.M., \& Perrett, D.I. (2001). Symmetry, sexual dimorphism in facial proportions and male facial attractiveness. Proceedings of the Royal Society B-Biological Sciences. 268, 1617-1623.

Perrett, D. I., Lee, K. J., Penton-Voak, I., Rowland, D., Yoshikawa, S., Burt, D. M., Henzl, S. P., Castles, D. L. \& Akamatsu, S. (1998). Effects of sexual dimorphism on facial attractiveness. Nature, $394,884-887$.

Pound, N., Penton-Voak, I. S., \& Surridge, A. K. (2009). Testosterone responses to competition in men are related to facial masculinity. Proceedings of the Royal Society B-Biological Sciences, 276, 153-159.

Puts, D.A. (2010). Beauty and the beast: mechanisms of sexual selection in humans. Evolution and Human Behavior, 31, 157-175.

Stirrat, M., \& Perrett, D. I. (2010). Valid facial cues to cooperation and trust: Male facial width and trustworthiness. Psychological Science, 21, 349-354.

Stirrat, M., \& Perrett, D. I. (in press). Face structure predicts cooperation: men with wider faces are more generous to their in-group when out-group competition is salient. Psychological Science.

Stirrat, M., Stulp, G., \& Pollet, T. V. (in press). Male facial width is associated with death by contact violence: Narrow-faced 
males are more likely to die from contact violence. Evolution and Human Behavior.

Tiddeman, B., Burt, D. M., \& Perrett, D. (2001). Prototyping and Transforming Facial Textures for Perception Research. IEEE Computer Graphics and Applications, 21, 42-50.

Weston, E.M., Friday, A.E., \& Lio, P (2007). Biometric Evidence that Sexual Selection Has Shaped the Hominin Face. PLoS ONE, 2, e710. 